\title{
Factors influencing local attitudes towards the conservation of leopard cats Prionailurus bengalensis in rural Taiwan
}

\author{
I A N Best and Kurtis Jai-Chyi Pei
}

\begin{abstract}
Understanding the human dimension is critical for effective conservation management of species involved in human-carnivore conflict. There is also a need to recognize who among the local human population is supportive of wildlife conservation. We investigated how local people's attitudes and knowledge of the leopard cat Prionailurus bengalensis are influenced by socio-demographic variables, encounter rates and the nature of encounters with the felid in Taiwan. During June-August 2014 we interviewed 150 residents in Miaoli County in northern Taiwan, where the leopard cat is known to occur. More than half of the participants indicated they were supportive of leopard cat conservation. The majority of those who expressed positive attitudes towards protection of the leopard cat belonged to younger, more educated socio-demographic groups. Negative attitudes towards leopard cat conservation were most prevalent among farmers, who also reported the highest incidence of negative experiences, mainly involving predation of poultry. We provide recommendations to mitigate human-felid conflict, including changes to animal husbandry practices. We also describe how conservation efforts for this species and wildlife in general in Taiwan could be improved, for example through incentive and awareness-raising programmes.
\end{abstract}

Keywords Attitudes, felid conservation, human-carnivore conflict, leopard cat, socio-demographic factors, Taiwan, threatened species

Supplementary material for this article is available at https://doi.org/10.1017/So030605318000984

\section{Introduction}

ffective wildlife conservation planning and manage-

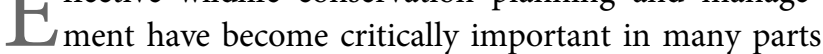
of the world, with more and more species threatened

IAN BEST* (Corresponding author) Department of Biology, Lund University, Lund, Sweden. E-mail ianbest03@gmail.com

KuRTIS JAI-Chy Pei (Corresponding author) Institute of Wildlife Conservation, College of Veterinary Medicine, National Pingtung University of Science and Technology, Neipu, Pingtung, Taiwan. E-mail kcjpei@gms.ndhu.edu.tw

${ }^{*}$ Also at: Biodiversity Program, Taiwan International Graduate Program, Academia Sinica, Taipei, Taiwan

Received 7 February 2018. Revision requested 16 April 2018

Accepted 16 July 2018. First published online 28 February 2019. and facing population declines (Ripple et al., 2014). Contemporary approaches to wildlife conservation have become more interdisciplinary, emphasizing the need for research that considers human dimensions and socioeconomic as well as ecological factors (Marchini, 2014; Verdade et al., 2014). In areas where people and carnivores increasingly come into contact as human activities encroach on wildlife habitat, an understanding of human-carnivore interactions and underlying human-human conflicts is essential (Young et al., 2010; Redpath et al., 2015), and opinions and attitudes toward carnivores will undoubtedly vary (Jacobs et al., 2012; Suryawanshi et al., 2014).

The leopard cat Prionailurus bengalensis is categorized as Least Concern on the IUCN Red List (Ross et al., 2015) but on the island of Taiwan it is categorized as Endangered and is protected under Taiwan's Wildlife Conservation Act. Leopard cats were once widely distributed throughout Taiwan but are now limited to Miaoli County, Taichung City and Nantou County (Pei, 2008; Chen et al., 2016). Their current habitats, in low-elevation mountainous regions with secondary vegetation, are in close proximity to human settlements and agricultural areas and are thus vulnerable to further development and encroachment (Pei, 2008; Chen et al., 2016). The leopard cat population on the island is estimated to comprise c. 500 individuals; a small fraction of the former population (Pei et al., 2014). The decline has been caused by anthropogenic factors, including commercial hunting for pelts, and habitat loss, degradation and fragmentation (McCullough, 1974; Ross et al., 2015). Regardless of the leopard cat's protected status under the Wildlife Conservation Act, illegal hunting still takes place (Pei, 2008; St. John et al., 2015). With increased infrastructure development and habitat fragmentation, leopard cats are also occasionally killed by motorized vehicles while attempting to cross roads (Pei et al., 2014).

Conflicts between people and leopard cats in Taiwan are often a result of poultry predation incidents, and sometimes lead to retaliatory killings of leopard cats by trapping or poisoning. In a survey conducted in Miaoli County (St. John et al., 2015), $6-9 \%$ of 242 respondents $(n=242)$ admitted to killing leopard cats during 2009-2012. Conflicts and negative attitudes are likely to persist if farmers' livelihoods are threatened and they suffer economic losses, especially in the case of small-scale poultry farmers (St. John et al., 2015). Human-human conflicts also occur in Taiwan between groups with varying interests concerning leopard cat conservation 


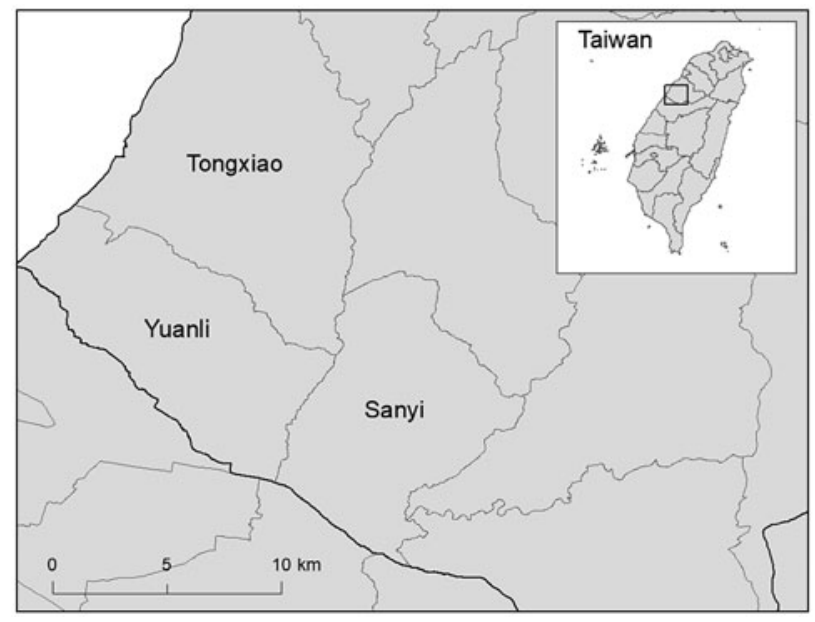

FIG. 1 Locations of the townships Tongxiao, Yuanli and Sanyi in Miaoli County, Taiwan, where surveys were conducted to investigate local attitudes to leopard cats Prionailurus bengalensis.

(Pei et al., 2014). For example, in Miaoli County there have been disputes between those interested in preserving leopard cat habitat and those with other interests, such as development projects. The majority of leopard cat hotspots (quality habitat with higher density of the species) occur on privately owned land (Pei et al., 2014), and therefore the success of leopard cat conservation efforts depends on the support of private landowners.

The objectives of this exploratory study were to investigate how attitudes and awareness of leopard cat conservation are influenced by various socio-demographic factors in Miaoli County, northern Taiwan. We also determined the frequency and nature of encounters, and whether they contribute to human-carnivore conflict and affect attitudes towards conservation. We predicted that (1) any prior negative experiences involving leopard cats, such as poultry predation, would influence participants' attitudes, and (2) individuals that had participated in any conservation activities would have more favourable attitudes toward the conservation of leopard cats. We provide novel data on local people's attitudes toward the conservation of a threatened carnivore, and contribute to improved understanding of the human dimensions of wildlife conservation in a rapidly developing East Asian country.

\section{Study area}

We conducted the study in three townships in Miaoli County: Sanyi, Tongxiao and Yuanli (Fig. 1). These locations were chosen based on the latest data available for the distribution of leopard cats, and hotspots for the species (Pei et al., 2014; Chen et al., 2016). We expected that local residents in these townships would have a greater chance of encountering leopard cats. Furthermore, $45 \%$ of farmers in this region are engaged in small-scale poultry farming for personal use (Mai, 2013). Also, 67\% of the land in Miaoli County is secondary forest, artificial forest, bamboo forest or agricultural land (Miaoli County Government, 2018), which can be suitable habitat for leopard cats (Pei et al., 2014; Ross et al., 2015).

\section{Methods}

We conducted a social survey utilizing a semi-structured questionnaire (Supplementary Table 1) during 5 July-28 August 2014. The questionnaire contained both closedand open-ended questions. The questionnaire was administered during one-to-one interviews designed to elicit information concerning socio-demographic factors (including age, gender, education level and occupation), attitudes and perceptions regarding leopard cats, knowledge of leopard cat ecology and conservation, prior experiences with the felid, and experience with conservation organizations and/or groups. These socio-demographic variables are commonly used in studies of attitudes to wildlife conservation (Kideghesho et al., 2007; Kansky \& Knight, 2014; Mkonyi et al., 2017).

We carried out preliminary tests of the questionnaire on colleagues and made any necessary revisions. We interviewed 150 local residents (50 per township), accounting for c. $2.5 \%$ of households. The majority of interviews were conducted in Mandarin, with a few in English and some in Taiwanese; an interpreter was present during all interviews. Each interview lasted 10-30 minutes and all were recorded, with the permission of the participants. All interviews were later transcribed into English. The same researcher (IB) facilitated the questionnaire in all interviews.

All participants were 18 years of age or older. Before the interview, participants were shown a picture of a leopard cat and if they were familiar with the study species they were asked to complete the questionnaire.

We utilized a combination of snowball and random sampling. Snowball sampling techniques is effective for facilitating access to populations or interest groups that may be challenging to reach (Atkinson \& Flint, 2001). We employed this technique to gain access to local people whose land overlaps leopard cat habitat, those who had lost poultry to leopard cat depredation, and individuals known locally to have hunting experience. In the centre of each township we chose locations at random using a random number generator, to recruit interviewees. To avoid bias we did not interview more than one member of any household.

Based on the information collected in the interviews, participants were categorized as either supportive or neutral/ unsupportive with respect to their attitudes towards leopard cat protection (Table 1). The strict conditions for the supportive group were to control for an over-representation 
TABLE 1 Key for assigning interviewees to groups based on their attitudes to leopard cat Prionailurus bengalensis protection and their knowledge of leopard cats and their ecology.

\begin{tabular}{ll}
\hline Questions & Responses \\
\hline Leopard cat protection & \\
$\begin{array}{l}\text { (1) Do you think protecting leopard cats is more important } \\
\text { than development projects in the region? }\end{array}$ & 'Yes' to all three questions = Supportive group \\
(2) Would you be willing to help protect leopard cats? & \\
(3) If there were leopard cats living on your land would you & \\
tolerate/protect them? & \\
Knowledge of leopard cats and their ecology & All questions answered correctly = High group \\
(1) What is suitable habitat for leopard cats? & $2-3$ questions answered correctly = Medium group \\
(2) How many leopard cats can be found in Miaoli? & $0-1$ questions answered correctly = Low group \\
(3) What does a leopard cat diet consist of? & \\
(4) How much land does one leopard cat need? & \\
\hline
\end{tabular}

TABLE 2 Summary statistics of $\chi^{2}$ tests to determine associations between the dependent variables 'support for leopard cat protection' and 'knowledge'.

\begin{tabular}{|c|c|c|c|c|c|c|c|c|}
\hline \multirow[b]{2}{*}{ Variable } & \multicolumn{4}{|c|}{ Support for leopard cat protection } & \multicolumn{4}{|c|}{ Knowledge } \\
\hline & $n^{1}$ & df & $\chi^{2}$ & $\mathrm{P}$ & $\mathrm{n}^{1}$ & df & $\chi^{2}$ & $\mathrm{P}$ \\
\hline$\overline{\text { Age }}$ & 141 & 1 & 8.24 & $0.004^{* * *}$ & 141 & 2 & $2.80^{2}$ & 0.238 \\
\hline Gender & 150 & 1 & 3.32 & 0.081 & 150 & 2 & 8.38 & $0.012^{*}$ \\
\hline Education level & 149 & 4 & 15.11 & $0.004^{* * *}$ & 149 & 8 & $4.61^{2}$ & 0.799 \\
\hline Occupation & 150 & 8 & 24.12 & $0.002^{* * *}$ & 150 & 16 & $13.89^{2}$ & 0.494 \\
\hline Experience $^{3}$ & 149 & 1 & 1.90 & 0.168 & 149 & 2 & 1.56 & 0.495 \\
\hline Experience type ${ }^{4}$ & 56 & 1 & 5.75 & 0.016 & 56 & 2 & $0.74^{2}$ & 0.793 \\
\hline Conservation experience ${ }^{5}$ & 149 & 1 & 6.26 & $0.012^{\star}$ & 149 & 1 & $8.26^{2}$ & $0.012^{\star}$ \\
\hline
\end{tabular}

${ }^{1}$ Varies because not all respondents answered all questions.

${ }^{2} \chi^{2}$ statistic calculated with Fisher's exact test (used when the subgroup sample of a variable was $<5$ ).

${ }^{3}$ Previous experiences with leopard cats: yes or no.

${ }^{4}$ Type of experience with leopard cats: neutral or negative.

${ }^{5}$ Previous experiences with conservation activities: yes or no.

${ }^{*} \mathrm{P}<0.05,{ }^{* * *} \mathrm{P}<0.005$.

of participants in this group and acquire a more realistic estimation of supportive individuals in our sample. We also defined conditions for groups reflecting their level of knowledge (high, medium or low) of leopard cat ecology and current conservation status (Table 1).

Participants were also categorized according to whether or not they had encountered leopard cats, and whether their encounters had been negative or neutral. For example, depredation of an interviewee's poultry by a leopard cat would qualify as a negative experience. Additionally, we determined if participants had any previous experiences with conservation organizations, groups and/or activities (denoted the conservation experience variable). Our explanatory variables were derived from the socio-demographic and experience factors, and our dependent variables were leopard cat protection, and knowledge.

We used SPSS v. 22.o (IBM, Armonk, USA) for statistical analyses. Descriptive statistical tests were used to compute cross-tabulations, and $\chi^{2}$ tests to determine association and significance between the two dependent variables
(Huizingh, 2007). Fisher's exact tests were used when required for quality control (Upton, 1992).

\section{Results}

Of the 150 participants, $53.3 \%$ were supportive of leopard cat protection (Supplementary Table 2). Furthermore, the majority of the participants considered leopard cats to be important $(85.3 \%)$, more than half perceived them positively $(54.0 \%)$, and more than half considered protecting leopard cats to be more important than further development in the region (57.3\%). The majority of the participants $(56.7 \%)$ had a medium level of knowledge about leopard cats; only 3.3\% had a high level of knowledge.

Individuals with a higher level of education were more supportive of leopard cat protection (Table 2, Fig. 2b), and participants younger than 45 years were more supportive than those 45 or older (Table 2, Fig. 2a). Farmers had the highest opposition (neutral/unsupportive) to leopard cat 

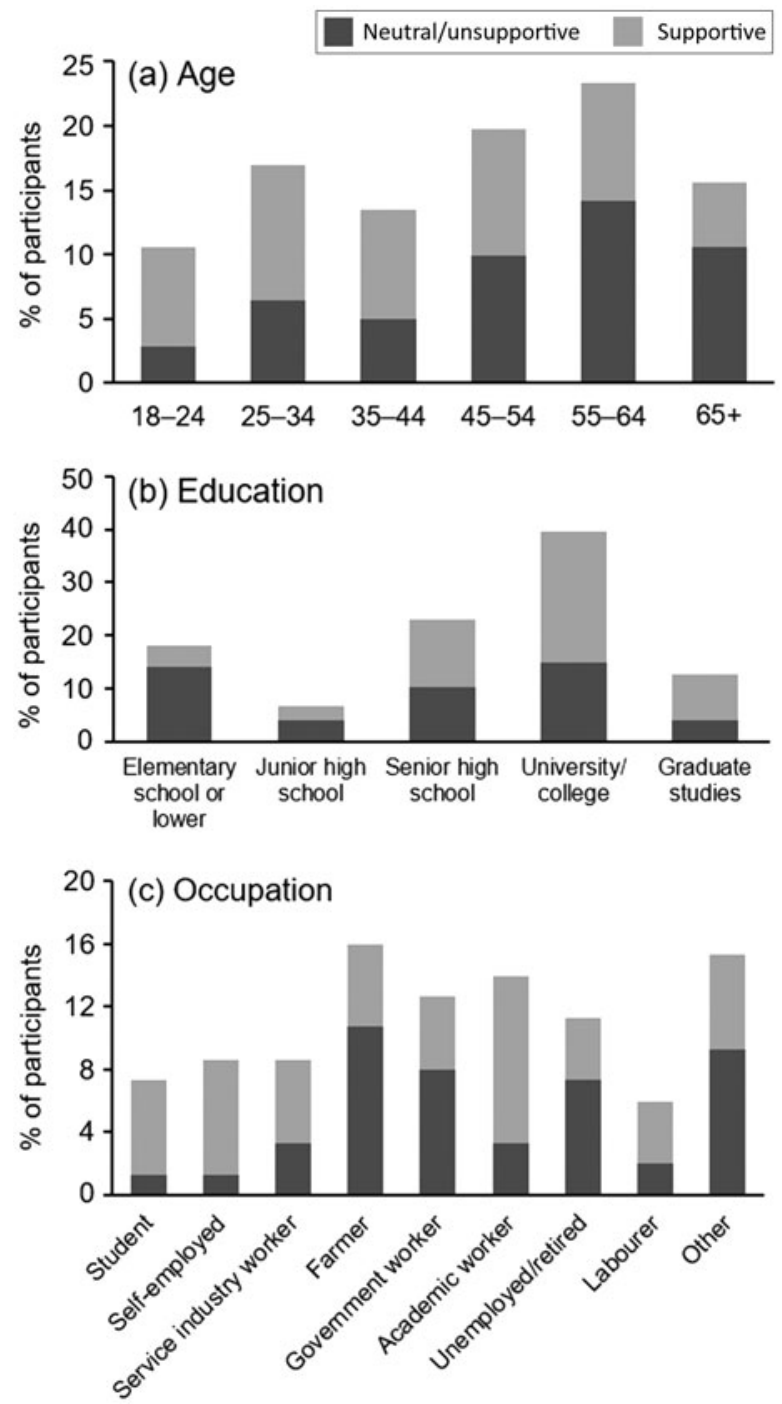

FIG. 2 Attitudes towards leopard cat protection, based on (a) age $(n=141)$, (b) education level $(n=149)$ and $(c)$ occupation of 150 interviewees in three townships in Miaoli County, Taiwan (Fig. 1).

protection compared to other occupational groups (Table 2, Fig. 2c).

We found that gender did not affect attitudes towards leopard cat protection. However, for the variable 'knowledge', the only significant association was with gender: there was a higher proportion of male participants in the medium knowledge group (Table 2).

Less than half of the participants (37.6\%) had personal experiences/encounters with leopard cats. The majority of those experiences were sightings but 15 ( $26.8 \%$ of 56$)$ were defined as negative experiences (mainly poultry depredation incidents $(86.7 \%)$, the remainder being attributed to hunting of leopard cats, which was information freely divulged by the participants).

Whether or not participants had had a personal experience with leopard cats did not influence their attitudes toward leopard cat conservation, or their level of knowledge
(Table 2). However, participants who reported negative experiences had a higher proportion in the neutral/unsupportive group (Table 2, Fig. 3a). Individuals who selfidentified as farmers not only had the highest incidence of experiences with leopard cats but also of negative experiences with leopard cats, compared with other occupational groups; nine out of 20 experiences with leopard cats reported by this occupational group were negative (Fig. 3b). Farmers who reported having had negative experiences all claimed to have small-scale poultry farms. The other participants (not in the farmers group) who reported having had negative experiences with leopard cats as a result of poultry predation stated during the interviews that they were relatives of poultry farmers or had economic interests in a farm.

Only $31.5 \%$ of 149 individuals claimed to have participated in some sort of conservation activity through an organization or group. These participants were more supportive of leopard cat protection and more aware of the ecology and status of the leopard cat (Table 2).

\section{Discussion}

More than half of the participants were supportive of leopard cat conservation, stating that the species' habitat should be preserved and that they would be willing to help improve awareness of its current status and take part in promotional and/or educational activities supporting its conservation.

The attitudinal variable for leopard cat protection was associated with the socio-demographic variables education level and occupation. Participants with higher levels of education were more supportive of leopard cat protection. Positive attitudes towards wildlife conservation have been reported to increase with higher levels of education (Casey et al., 2005; Mkonyi et al., 2017). With respect to occupation, participants in the academic group were most frequently supportive, whereas farmers were most frequently neutral/ unsupportive. This is consistent with other surveys that showed farmers have more negative attitudes towards carnivores than other occupational groups (Hemson et al., 2009; Liu et al., 2011). Furthermore, as predicted, we found a strong association between negative experiences with leopard cats (almost entirely involving poultry predation incidents) and unsupportive and negative attitudes towards the felid and its protection. Those who have experienced conflict with carnivores are less likely to base their decisions, and subsequent actions, regarding wildlife conservation on objective facts or knowledge, as experiences can lead to emotional prejudice and subjectivity (Inskip \& Zimmerman, 2009; Slagle et al., 2012). However, we found the incidence of negative experiences with leopard cats to be relatively low, consistent with the findings of St. John et al. (2015), whose results showed that only 10 of 242 respondents surveyed 
(a) Attitudes towards protection

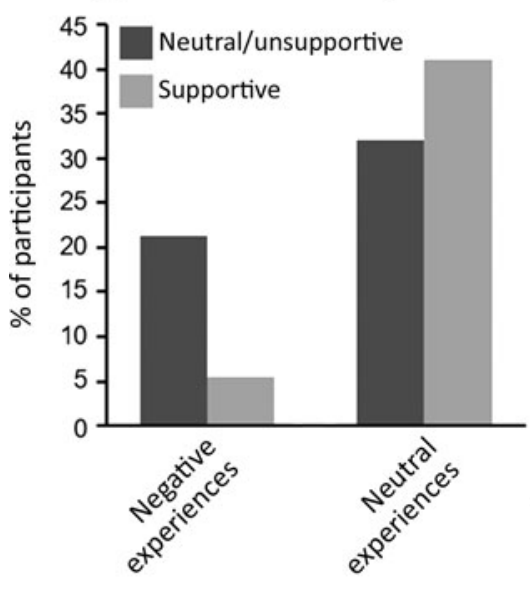

(b) Experience type

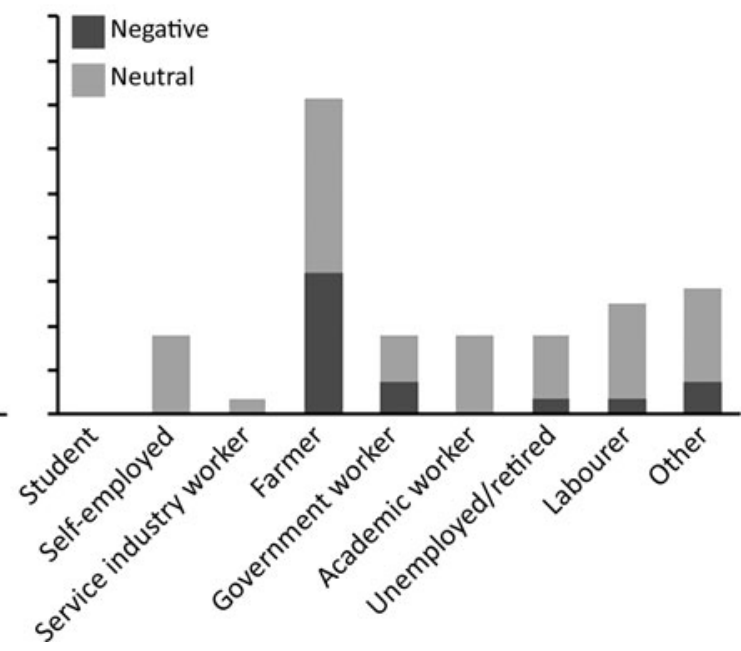

FIG. 3 (a) Attitudes towards leopard cat protection, based on experience type $(n=56)$, and (b) experience type within occupational subgroups $(\mathrm{n}=56)$ among interviewees in three townships in Miaoli County, Taiwan (Fig. 1). perceived leopard cats as pests, and all 10 owned small-scale poultry farms. The relatively low incidence of negative experiences and associated attitudes should not be overlooked however, because of the potential harm to the felid's reputation (Sakurai et al., 2013).

In terms of the relationship between age and supportiveness of leopard cat conservation, the results are significant when the participants are divided into two groups: $<45$ and $\geq 45$ years old. Other authors have reported that age is negatively associated with attitude towards carnivores; i.e. younger people have more positive attitudes towards these species (Casey et al., 2005; Suryawanshi et al., 2014).

Our results also indicate that conservation experience contributes to favourable attitudes towards leopard cats, which confirms our second prediction. This is not surprising, as we would expect individuals with conservation experience to have an interest in the subject and be more empathetic towards wildlife, especially threatened wildlife (Teel \& Manfredo, 2009). Individuals with conservation experience had higher levels of knowledge of leopard cat ecology and status.

Few of the participants were able to correctly answer the question pertaining to the current status of leopard cats, which implies that awareness could be improved at the local level. St. John et al. (2015) found that few of the respondents in their survey were aware that leopard cats are protected by law. Thus there is room for improvement in community-level environmental educational programmes in Taiwan (Hsu et al., 1997).

Awareness of and support for leopard cats could be increased by targeting younger, higher-educated sociodemographic groups through social media and other online platforms. Online sampling and outreach is becoming more widely used in the scientific community (Baltar \& Brunet, 2012). When asked how they would be willing to help support leopard cats, many of the participants in our study indicated they would be willing to help improve awareness. This could be achieved via online media platforms.

The success of conservation action plans for wildlife, especially carnivores, depends on the support of local people. For leopard cats, mitigation of any sources of conflict is a priority, especially as many farmers in Miaoli stock poultry. One of the participants in our study mentioned that he had installed powerful lights surrounding the enclosure of his poultry to deter leopard cats, which he claimed was successful. Lights can act as disruptive visual stimuli for predators, and have been found to be useful (Shivik \& Martin, 2001). Another effective, non-lethal management technique is to construct and/or strengthen and reinforce enclosures to maintain barriers between carnivores and livestock or poultry (Shivik \& Martin, 2001; Ravenelle \& Nyhus, 2017). Apart from alterations to animal husbandry, compensation schemes are commonly adopted to mitigate the impact of depredation (Zabel \& Holm-Muller, 2008; Maheshwari et al., 2014). However, in Taiwan ex-post compensation schemes are ineffective as they are not offered through the government but through NGOs, with minimal compensation, requirement for substantial evidence of losses, and lengthy processing time (Pei et al., 2014). Moreover, farmers may be reluctant to report losses to the government or conservation groups, as they may prefer to take matters into their own hands and carry out retaliatory killings of leopard cats (Kao, 2013).

As an alternative, programmes based on conservation performance payments have been designed and implemented in developed countries (e.g. Sweden) to support carnivore conservation, and these have been largely successful (Persson et al., 2015). Performance payments are made to individuals and/or groups for specific conservation achievements, such as successful reproduction of target species (Zabel \& Holm-Muller, 2008; Persson et al., 2015). The payments are contingent on reciprocity; i.e. the amount paid is dependent on specific conservation targets being met 
(Zabel \& Engel, 2010; Ravenelle \& Nyhus, 2017). Currently there are no incentives for private landowners in Taiwan to endorse leopard cat conservation but performance payment programmes could potentially be an effective means of mitigating impacts and promoting coexistence between leopard cats and landowners. A feasible approach for implementing such a programme could involve paying farmers and other landowners to record occurrences of mature and/or juvenile leopard cats in their habitat and hotspot areas. The conservation outcomes could be verified by trained scientists, and landowners would have an incentive to not only tolerate but also protect leopard cats.

Our findings indicated an appreciation for leopard cats amongst the residents of our three study areas. As leopard cats are the sole remaining wild, native felid species in Taiwan, their preservation is critical. However, leopard cats are threatened locally, and face population declines. To rectify this, conservation action plans need to be developed and implemented, with channels of discussion established between stakeholders, policymakers and conservation biologists to facilitate the necessary changes.

Acknowledgements We thank all those who participated in the interviews; Mei-Ting Chen, Hong-Wei Fong, Angel Lin and Tsai-Yu Lee for their assistance in arranging interviews in Miaoli; local academics for their assistance in revising our questionnaire; the translators for transcribing the interviews; Ola Olsson for his counsel and support of this study; and the Department of Biology, Lund University, Lund, Sweden for funding.

Author contributions Study development and writing: IB and KCJP; interviews and data analysis: IB.

\section{Conflicts of interest None.}

Ethical standards The authors abided by the Code of Conduct for contributors to Oryx, and the interview process and methodology conformed to the guidelines of the British Sociological Association. Approval was received from all participants to report the data collected from the personal interviews; all participants remain anonymous, to protect confidentiality.

\section{References}

Atkinson, R. \& Flint, J. (2001) Accessing hidden and hard-to-reach populations: snowball research strategies. Social Research Update, 33, $1-4$.

Baltar, F. \& BRUnet, I. (2012) Social research 2.o: virtual snowball sampling method using Facebook. Internet Research, 22, 57-74.

Casey, A.L., Krausman, P.R., Shaw, W.W. \& Shaw, H.G. (2005) Knowledge of and attitudes toward mountain lions: a public survey of residents adjacent to Saguaro National Park, Arizona. Human Dimensions of Wildlife, 10, 29-38.

Chen, M.-T., Liang, Y.-J., Kuo, C.-C. \& Pei, K.J.-C. (2016) Home ranges, movements and activity patterns of leopard cats (Prionailurus bengalensis) and threats to them in Taiwan. Mammal Study, 41, 77-86.

Hemson, G., Maclennan, S., Mills, G., Johnson, P. \& Macdonald, D. (2009) Community, lions, livestock and money: a spatial and social analysis of attitudes to wildlife and the conservation value of tourism in a human-carnivore conflict in Botswana. Biological Conservation, 142, 2718-2725.

Hsu, M.-J., Agoramoorthy, G., Desender, K., Baert, L. \& Bonilla, H.R. (1997) Wildlife conservation in Taiwan.

Conservation Biology, 11, 834-838.

Huizingh, E. (2007) Applied Statistics with SPSS. Sage, London, UK. Inskip, C. \& ZimmermanN, A. (2009) Human-felid conflict: a review of patterns and priorities worldwide. Oryx, 43, 18-34.

Jacobs, M.H., Fehres, P. \& Campbell, M. (2012) Measuring emotions towards wildlife: a review of generic methods and instruments. Human Dimensions of Wildlife, 17, 233-247.

Kansky, R. \& Knight, A.T. (2014) Key factors driving attitudes towards large mammals in conflict with humans. Biological Conservation, 179, 93-105.

KAO, C.-T. (2013) Removal patterns and conflicts of leopard cats (Prionailurus bengalensis chinensis) at Tongsiao Miaoli, Taiwan. MSc thesis. Institute of Wildlife Conservation, National Pingtung University of Science and Technology, Pingtung, Taiwan. [In Chinese, with English abstract]

Kideghesho, J.R., Røskaft, E. \& Kaltenborn, B.P. (2007) Factors influencing conservation attitudes of local people in Western Serengeti, Tanzania. Biodiversity and Conservation, 16, 2213-2230.

Liu, F., McShea, W.J., Garshelis, D.L., Zhu, X., WanG, D. \& Shao, L. (2011) Human-wildlife conflicts influence attitudes but not necessarily behaviors: factors driving the poaching of bears in China. Biological Conservation, 144, 538-547.

Maheshwari, A., Midha, N. \& Cherukupalli, A. (2014) Participatory rural appraisal and compensation intervention: challenges and protocols while managing large carnivore-human conflict. Human Dimensions of Wildlife, 19, 62-71.

MAI, C.-H. (2013) Current status of small carnivores hunting in rural area of Miaoli, Taiwan. MSc thesis. Institute of Wildlife Conservation, National Pingtung University of Science and Technology, Pingtung, Taiwan. [In Chinese, with English abstract]

Marchini, S. (2014) Who's in conflict with whom? Human dimensions of the conflicts involving wildlife. In Applied Ecology and Human Dimensions in Biological Conservation (eds L.M. Verdade, M.C. Lyra-Jorge \& C.I. Pina), pp. 189-210. Springer, New York, USA.

McCullough, D.R. (1974) Status of Larger Mammals in Taiwan. A Report to the World Wildlife Fund. Tourism Bureau, Taipei, Taiwan.

Miaoli County Government (2018) Regional Planning. Http:// landev.miaoli.gov.tw/mi/po2-2.html [accessed 28 May 2018].

Mkonyi, F.J., Estes, A.B., Msuha, M.J., Lichtenfeld, L.L. \& Durant, S.M. (2017) Local attitudes and perceptions toward large carnivores in a human-dominated landscape of Northern Tanzania. Human Dimensions of Wildlife, 22, 314-330.

PEI, K.J.-C. (2008) Present status and conservation of small carnivores at low elevation mountains in Hsinchu County and Miaoli County (3/3). Final report, In Forestry Bureau Cons. Res. 96-01, p. 88. Forestry Bureau, Taipei, Taiwan. [In Chinese, with English abstract] Pei, K.J.-C., Lu, D.-J., Hwang, M.-H., Chao, J.-L., Chen, M.-T. (2014) Initiating community-involved conservation activities for endangered leopard cats in Miaoli, Taiwan. Final report. In Forestry Bureau Cons. Res. 100-02-08-02, p. 72. Forestry Bureau, Taipei, Taiwan. [In Chinese, with English abstract]

Persson, J., Rauset, G.R. \& Chapron, G. (2015) Paying for an endangered predator leads to population recovery. Conservation Letters, 8, 345-350.

Ravenelle, J. \& Nyhus, P.J. (2017) Global patterns and trends in human-wildlife conflict compensation. Conservation Biology, 31, 1247-1256.

Redpath, S.M., Bhatia, S. \& Young, J. (2015) Tilting at wildlife: reconsidering human-wildlife conflict. Oryx, 49, 222-225. 
Ripple, W.J., Estes, J.A., Beschta, R.L., Wilmers, C.C., Ritchie, E. G., Hebblewhite, M. et al. (2014) Status and ecological effects of the world's largest carnivores. Science, 343, 1241484.

Ross, J., Brodie, J., Cheyne, S., Hearn, A., Izawa, M., Loken, B. et al. (2015) Prionailurus bengalensis. In The IUCN Red List of Threatened Species 2015: e.T18146A50661611. Http://dx.doi.org/10. 2305/IUCN.UK.2015-4.RLTS.T18146A50661611.en [accessed 11 January 2017].

Sakurai, R., Jacobson, S. K. \& Ueda, G. (2013) Public perceptions of risk and government performance regarding bear management in Japan. Ursus, 24, 70-82.

Shivik, J.A. \& Martin, D.J. (2001) Aversive and disruptive stimulus applications for managing predation. Wildlife Damage Management Conference, 9, 111-119.

Slagle, K.M., Bruskotter, J.T. \& Wilson, R.S. (2012) The role of affect in public support and opposition to wolf management. Human Dimensions of Wildlife, 17, 44-57.

St. John, F.A.V., MaI, C.H. \& Pei, K.J.-C. (2015) Evaluating deterrents of illegal behaviour in conservation: carnivore killing in rural Taiwan. Biological Conservation, 189, 86-94.

Suryawanshi, K.R., Bhatia, S., Bhatnagar, Y.V., Redpath, S. \& Mishra, C. (2014) Multiscale factors affecting human attitudes toward snow leopards and wolves. Conservation Biology, 28, 1657-1666.

Teel, T.L. \& Manfredo, M.J. (2009) Understanding the diversity of public interests in wildlife conservation. Conservation Biology, 24, 128-139.

Upton, G. J. (1992) Fisher's exact test. Journal of the Royal Statistical Society. Series A (Statistics in Society), 155, 395-402.

Verdade, L.M., Pina, C.I. \& Lyra-Jorge, M.C. (2014) Redirections in conservation biology. In Applied Ecology and Human Dimensions in Biological Conservation (eds L.M. Verdade, M.C. Lyra-Jorge \& C.I. Pina), pp. 3-17. Springer, New York, USA.

Young, J.C., Marzano, M., White, R.M., McCracken, D.I., Redpath, S.M., Carss, D.N. et al. (2010) The emergence of biodiversity conflicts from biodiversity impacts: characteristics and management strategies. Biodiversity and Conservation, 19, 3973-3990.

ZABEL, A. \& ENGEL, S. (2010) Performance payments: a new strategy to conserve large carnivores in the tropics? Ecological Economics, 70, 405-412.

Zabel, A. \& Holm-Múller, K. (2008) Conservation performance payments for carnivore conservation in Sweden. Conservation Biology, 22, 247-251. 